\title{
THERMAL FLOW MICRO SENSORS ${ }^{1}$
}

\author{
M. Elwenspoek \\ MESA Research Institute, University of Twente, P.O.Box 217, \\ 7500 AE Enschede, The Netherlands \\ email: m.elwenspoek@el.utwente.nl
}

\begin{abstract}
A review is given on sensors fabricated by silicon micromachining technology using the thermal domain for the measurement of fluid flow. Attention is paid especially to performance and geometry of the sensors. Three basic types of thermal flow sensors are discussed: anemometers, calorimetric flow sensors and time of flight flow sensors. Anemometers may comprise several heaters and temperature sensors and from a geometric point of view are similar sometimes for calorimetric flow sensors. We find that depending on the Reynolds number to or three element anemometers may perform as calorimetric sensors (very small Re) while calorimetric flow sensors operate like anemometers in the high Re regime.
\end{abstract}

\section{INTRODUCTION}

Thermal flow sensors are transducers, which comprise heaters and temperature sensors. The heat interacts with the surrounding: a streaming liquid carries away some heat and the temperature distribution around the heater changes in conjunction with the moving fluid. In particular, the temperature of the heater depends on the flow. Silicon micromachining allows the fabrication of small components where many different functions (e.g. arrays of temperature sensors and heaters, pressure sensors, shear stress sensors, etc.) can be integrated so that the functionality of the sensors can be increased. Further more, due to the small size of the elements these sensors can be quite fast. Speed is important in turbulent flow and in acoustics.

We can classify thermal flow sensors in three basic categories:

- Anemometers

- Calorimetric flow sensors

- Time of flight sensors

Generally, anemometers comprise of a single element which is heated and the temperature of which is measured. Since the electric resistivity of most materials depends strongly on the temperature, and an electrical current heats the elements, the measurement of the resistivity of the element is a natural choice. Any flow will enhance the rate at which heat is transported away from the element. The anemometer can be operated in two modes: constant power and constant temperature. The simplest mode is the mode where the anemometer is fed with constant power, and the temperature of the hot element is measured. The response time in this mode is given by the heat capacity of the hot element and the rate at which heat is transferred to the medium (RC-time). When the temperature is held constant - this requires a feed back loop - the power needed to keep the temperature constant is measured. In this mode the anemometer is considerably faster than in the constant power mode.

The signal of the anemometer is proportional to the square root of the flow velocity as a consequence of the boundary layer.

\footnotetext{
${ }^{1}$ This paper is based on a part of a chapter on micro flow sensors in a book on mechanical microsensors to be published at Springer
}

$0-7803-5139-8 / 99 / \$ 10.00$ C 1999 IEEE 
Calorimetric flow sensors and time of flight sensors require two or more elements. A standard configuration consists of a heater surrounded by temperature sensitive elements arranged symmetrically downstream and upstream. Any flow will carry away heat in the direction of the flow and accordingly will cool down the heater and change the temperature distribution around it. The temperature difference upstream and downstream is measured. The signal (proportional to $\Delta T$ ) is proportional to the flow velocity close to $U=0$, but saturates and even decreases at higher flow velocity. Calorimetric flow sensors are useful at very low flow velocities.

In time of flight sensors a heatpulse is fed to the fluid and a temperature sensor located downstream detects its delay. Measuring a time, in this mode the sensors give truly the velocity of the streaming fluid.

We do not discuss a class of thermal flow sensors here, which are the skin friction sensors. We refer to Elwenspoek and Wiegerink (2000) for a recent overview.

\section{ANEMOMETER TYPE FLOW SENSORS}

In the following we describe and discuss briefly issues of design, fabrication, and performance of a few example for micro anemometers.

The traditional anemometer technology is based on the hot wire. Here a wire, typically with a length in the $\mathrm{mm}$ range and a thickness below $10 \mu \mathrm{m}$, is suspended between two holders. In order to make the sensor fast, the wire must be thin to minimize the heat capacity, and to make it sensitive, the heat conduction to the support must be as small as possible.

The basic characteristics are response time, sensitivity to magnitude and direction of flow.

Response Time

The response time of a hot wire is given by (see. e.g. Lomas 1986)

$$
f_{c}=\frac{P(U) R \alpha}{2 \pi \rho c A l} \frac{R}{\Delta R}
$$

with $P(U)$ the velocity dependent power fed to the hot wire. Typical response times are in the range of ms. When driving the anemometer with constant power, the response time can be larger by orders of magnitude (Freymuth 1977).

In micro technology, the wire is replaced by a thin film deposited on a silicon substrate. In fact, first designs were fabricated in this way (van Riet 1976, van Putten 1974, Huysing 1980, Yang 1992). This has a disadvantage, which is immediately apparent: the heat capacity is the defined by the chip, and the heat conduction to the support is large due to the high thermal conductivity of silicon. Three different roads were went to solve these problems:

- The micromachined element carrying the resistor is thermally isolated from the rest of the structure using a material with low heat conductivity. An example of this approach has been presented by e.g. Stemme 1986, Löfdahl (1992), see fig. 1.

- The thin film resistor is placed on a membrane. This has the effect that heat diffusion is restricted to lateral diffusion in the thin membrane, and that the substrate supporting the membrane is more or less at the temperature of the fluid (the surrounding). Examples for this techniques (see fig. 2) are provided by van der Wiel (1993) and Jiang (1996), Ashauer (1998) and Kälvesten (1996).

- The thin film resistor is placed on top of micro bridges crossing a flow channel. This technique restricts the heat conduction to the substrate within a one-dimensional structure. Examples for this technique can be found in Urban (1990), Ebefors (1998), Kohl (1994), Lyons (1998), Stephan (1991), Lammerink (1993), van Kuik (1995). 


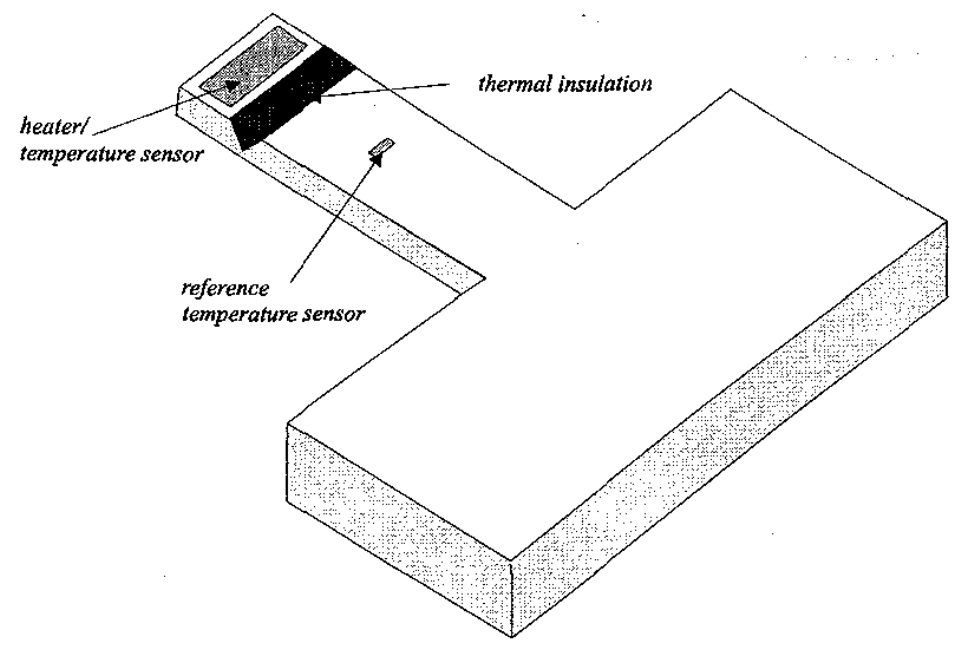

Fig. I In Stemme's micro-anemometer design (Stemme 1986) polyimide is used to isolate the hot part of the sensor from the support. Temperature sensors and heater elements are diodes

The MEMS group at ETH Zürich used a design somewhere between a membrane and a bridge: The structure of their flow sensors is a wide and thin cantilever beam (e.g. Funk (1993) Moser (1991), Mayer (1996), Wachutka (1991). Swart (1991) proposes a membrane suspended by four beams.

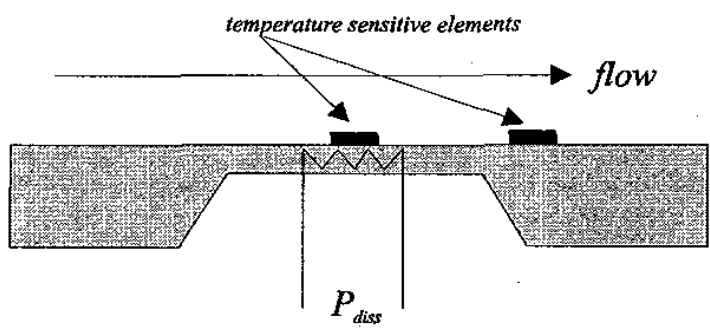

Fig. 2. Heater element on top of a membrane

Micromachined flow sensors are not much faster than the classical hot wire anemometer. Ebefors reports cooling and heating times of 0.1 and $0.3 \mathrm{~ms}$, respectively. The fastest sensors of de Bree et al. (de Bree 1995, 1996, 1997, 1998) have cutoff frequencies a little bit above $1 \mathrm{kHz}$. Also Lyons reports response times of not shorter than $2 \mathrm{~ms}$.

\section{Sensitivity}

The anemometers loose their heat to the fluid by forced convection and by heat diffusion to the support. The total heat flux therefore can be expressed as

$$
Q=(\alpha+\beta \sqrt{\operatorname{Re}}) \Delta T
$$

$\alpha$ represents the conductive heat flux to suipport and to the fluid, and $\beta$ to the streaming fluid due to convection. Anemometers must be designed so that $\alpha$ is minimized with respect to $\beta$.

Conductive heat flux to the support is minimized by minimizing the cross section of the thin film and its mechanical carrier and by choosing a material with low heat conduction. The first point shows that beams as support are expected to perform superior over membranes, and that a narrow beam is better than a wide beam. Silicon is a material with high thermal conductivity $(100 \mathrm{~W} / \mathrm{mK})$, while silicon nitride is one of the materials with the smallest thermal conductivity (see e.g. Sanchez et al. (1996)). 


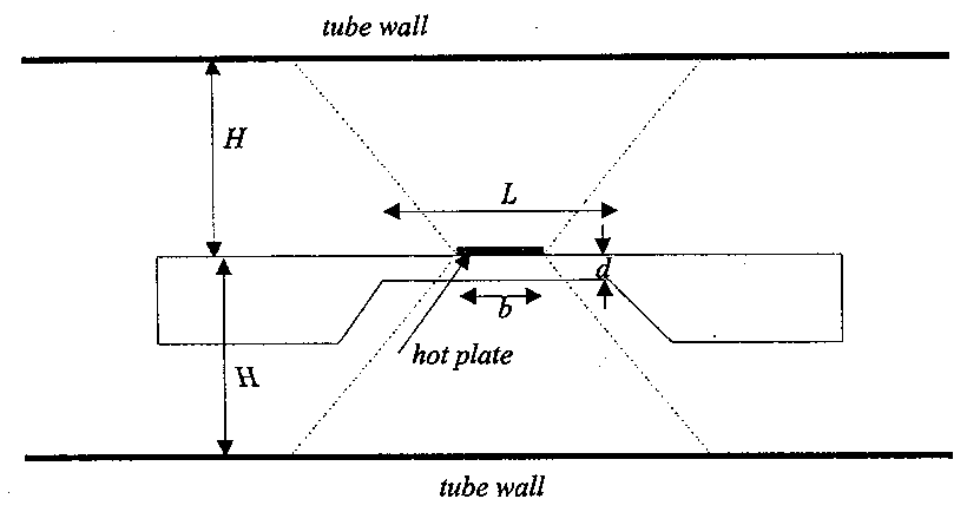

Fig. 3. Geometry of conductive heat transfer from a sensor chip to tube walls. The dashed lines indicate the cone through which heat flows according to the model.

Conductive heat flux to the fluid seems to be important if the sensor element is close to walls. As an example consider fig. 3. Fingerson and Freymuth (1996) mention that for hot wire anemometers, the distance to the wall must be 500 times larger than the diameter of the wire in order to eliminate the effect of heat conduction to the wall completely. Van der Wiel gives an estimate for the heat conduction from the sensor to the duct, by simplifying the geometry of conductive heat flow, as indicated in fig. 3. Van der Wiel's result (van der Wiel 1993) is

$$
Q=4 \lambda \sqrt{A}(1+\sqrt{A} / H) \Delta T
$$

with $H$ the cross-section of the tube and the chip in its center and $A$ is the area of the heater. For the heat loss to the substrate a similar geometry is assumed, which leads to

$$
Q=8 \lambda_{\text {substrate }} d / \ln (L / b) \Delta T \text {. }
$$

The meaning of the symbols is given in fig. 3. Note that the hydrodynamic equation are not linear, therefore solutions for the case that $U=0$ cannot be superimposed to solution with $U \neq 0$.

Kohl (1994) notes that the sensitivity to flow becomes zero if the flow is too small. In Kohl's set up, there is a bridge crossing a channel. The distance for the sensor to the channel wall is in the range of $80-150 \mu \mathrm{m}$, with a heater width of $50 \mu \mathrm{m}$ it is clear that the conductive heat transport is very important. Contrary to the claims of Fingerson and Freymuth (1996), the sensitivity to the flow drops to zero if the flow velocity becomes smaller than a critical value. Kohl's experimental set up was such that entrance effects may play an important role. The length of the duct used by them was only $9 \mathrm{~mm}$, while the entrance length $\sim 2 \mathrm{~mm} \times \mathrm{Re}_{\mathrm{ch}}$, with $\operatorname{Re}_{\mathrm{ch}}$ up to $10^{4}$; $2 \mathrm{~mm}$ is the wetted perimeter of the channel. This might be the reason why they find distinct deviations form King's law (King 1914). The threshold velocity is attributed to a competition of heat conduction and convective heat transport; the latter looses if there is no boundary layer (Elwenspoek and Wiegerink 2000).

Wachutka et al. (1991) describe two types of flow sensors: An anemometer and the second one operates with a bridge suspended over an anisotropically etched recess, carrying a heater and two temperature sensors up- and down stream, respectively. The second one has a similar geometry as the first one, the basic difference is that it is a bridge in place of a cantilever beam. This device has a thermopile (polysilicon/aluminum) on top with the hot end at the end of the bridge and the cold one on the substrate. The bridges have been made from a sandwich of field oxide, CVD oxide and silicon nitride with a total thickness of $2 \mu \mathrm{m}$. The length and width are in the range of a few $100 \mu \mathrm{m}$, with an aspect ratio in the order of $2-3$. The fabrication technology is described in detail in Moser (1991).

The sensors suffer substantially from heat conduction to the substrate and do not compare in performance to the designs of Stemme et al (1986), fig. 1, who integrated a thermal insulation into a cantilever beam.

In studying thermal shear stress sensors, Jiang et al. (1996) compared three geometry's to find the optimum one for the sensitivity. The result is that a vacuum cavity below a silicon nitride membrane carrying the a hot thin film element increases the sensitivity by a factor of 4 . In Jiangs work, the sensors are used for shear stress 
imaging in turbulent flow. For this purpose, the sensors are quite small (membranes in the size of $150 \mu \mathrm{m}$, width of the wire of $3 \mu \mathrm{m}$ ) and there were placed on rows of 25 sensors. This way a shear stress image can be obtained.

\section{Angular sensitivity}

The sensitivity of a hot wire anemometer depends on the orientation of the velocity vector with respect to the direction of the wire. For infinitely long wires, a wind parallel to the wire cannot carry away any heat, therefore in this case, we have

$$
Q=(\alpha+\beta|\cos \theta| \sqrt{\operatorname{Re}}) \Delta T
$$

The sensitivity is proportional to $|\cos \theta|$, with $\theta$ the angle between the normal on the wire and the flow direction. There is no sensitivity at all with respect to the side from which the flow comes. In the limit of a wire which is as long as thick, the angular sensitivity of course must vanish. Therefore, hot wire anemometers with a finite length have a sensitivity more like (Fingerson 1996)

$$
Q=\left(\alpha+\beta \sqrt{\cos ^{2} \theta+f \sin ^{2} \theta} \sqrt{\operatorname{Re}}\right) \Delta T
$$

with $f$ a phenomenologically determined constant, which only weakly depends on the Reynolds number. $f$ approaches zero if the wire is more than 250 times longer than thick. This angular sensitivity has been used by Ebefors (1998) to construct a microsensor for turbulence studies in which the vector flow velocity was the target.

\section{TWO WIRE ANEMOMETERS}

Early work on flow sensors with two temperature sensors on the chip can be found in Huising (1980), van Putten (1983), van Riet (1976), Rehn (1980), van Oudhuisden (1990 a and b) and Stephan (1991). The main purpose to use two elements is to get a differential signal to eliminate common disturbances, such as temperature drifts. It is clear that the ambient temperature changes the resistance of a thermistor, so the change in ambient temperature gives a signal without flow.

A second purpose is the following. Two wires can be used to measure the temperature difference between two points in the streaming liquid, when one of the points or both are heated. In this case there is a sensitivity to the direction of the flow, contrary to the single element anemometer. The signal changes the sign if the flow direction reverses and if the flow is normal to the line connecting the temperature sensors the signal due to convection is equal to zero.

If it is assumed that the convective heat transport from the two points still is dominated by the thermal boundary layer - the boundary layers are thinner closer to the leading edge of the sensor structure, see fig 4 and 5 , than at the far end, the temperature difference between the two points still will depend on $\sqrt{ } U$ : For the case in fig. 4 , the upstream temperature sensor is at the ambient temperature, so the downstream sensor functions basically like a single wire anemometer. For the case shown in fig. 4, we would have at the upstream side:

$$
Q=\left(\alpha+\beta / \delta_{1}\right)\left(T_{1}-T_{0}\right)
$$

and down stream: $\quad$.

$$
Q=\left(\alpha+\beta / \delta_{2}\right)\left(T_{2}-T_{0}\right) \quad \text {. }
$$

with $T_{0}$ the temperature of the liquid. If the heat dissipation is equal at both points, we have

$$
\Delta T=\left(T_{1}-T_{2}\right)=\frac{1}{\alpha^{\prime}+\beta^{\prime} \sqrt{U}}
$$

So the signal will have the characteristic square root $U$ dependence just as the single hot wire anemometer. This has been confirmed experimentally (van Riet 1976, van Putten 1974, van Putten 1983). 


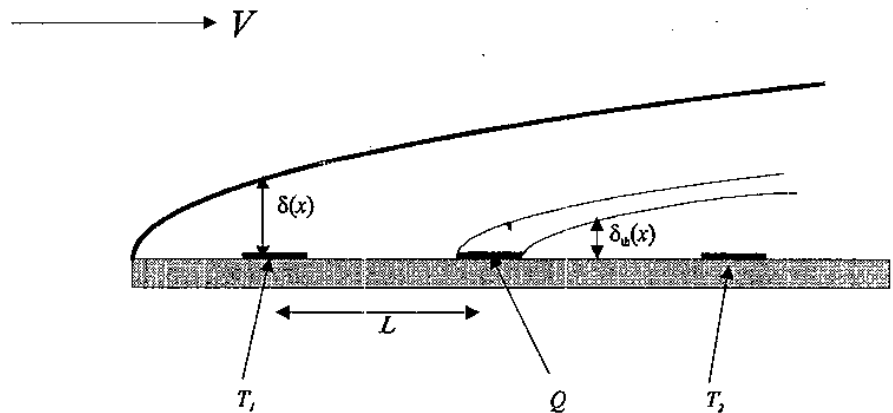

Fig. 4. Two wire anemometer. In this version, the heater is separated from the temperature sensors.

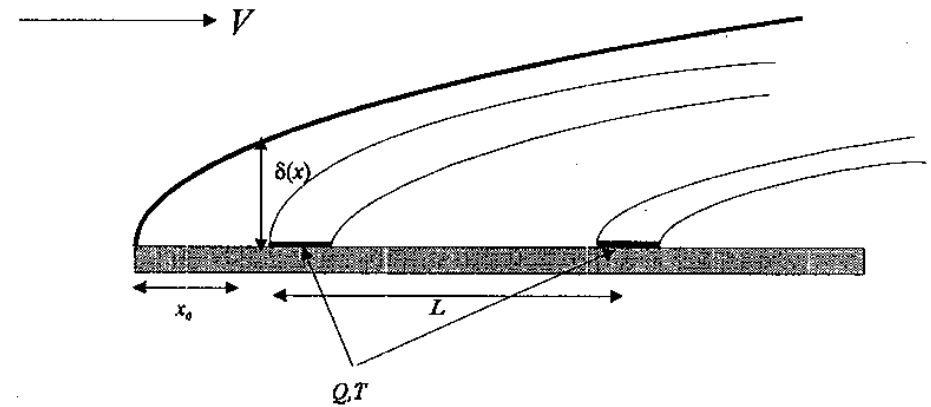

Fig. 5. A two wire anemometer on a single chip. In this version the heater and temperature sensors are physically the same structures

The boundary layer is dominant if its thickness is much smaller than the distance between the elements of the microstructure. If this is true, the heat loss is dominated by the heat diffusion over the boundary layer, and much less by heat diffusion between the elements. In the sensors described by van Riet (1976), the distance between the two elements is $l=0.2 \mathrm{~cm}$, and approximately equal to the streamwise length of the chip. The maximum thickness of the boundary layer is $\delta_{\max }=\sqrt{v l / U_{\min }}=500 \mu \mathrm{m}$ for $v_{\text {air }}=0.15 \mathrm{~cm}^{2} / \mathrm{s}$ (Landau 1974), $U_{\min } V_{\min } \sim$ $10 \mathrm{~cm} / \mathrm{s}$, considerably smaller than $l$. Analysis of the geometry used by van Oudhuisden $(1990 \mathrm{a}, \mathrm{b})$ and van Putten $(1974,1983)$ gives similar results: within the ranges studied experimentally we have always $\delta<<l$.

\section{CALORIMETRIC TYPE FLOW SENSORS}

Calorimetric flow sensors are constructed similar to the arrangements of heaters and temperature sensors as shown in fig.'s 4 and 5 . In most designs, calorimetric flow sensors comprise a heater with temperature sensors arranged symmetrically upstream and downstream with respect to the heater. The output signal is then the temperature difference at the sensors: the downstream sensor is heated, and the upstream sensor is cooled. Similar to the two element anemometers the direction of the flow can be determined by calorimetric flow sensors. A schematic of the temperature distribution is shown in fig. 6 . Commercial sensors have these elements mounted on the wall of the tube, most microsensors insert a chip carrying these elements into the flow or fabricate bridges across a micromachined flow channel. The former has the advantage of robustness and the disadvantage of slow speed and low sensitivity.

Basically, calorimetric flow sensors are mass flow sensors (see e.g. Hohenstatt 1990). The principal idea is to transfer some heat to a fluid, which is carried away by convection. So it is the amount of heat which is intended to measure, which is proportional to the mass flow. Note that the relation of the transported heat is dependent on the type of fluid: the specific heat is a important parameter. 


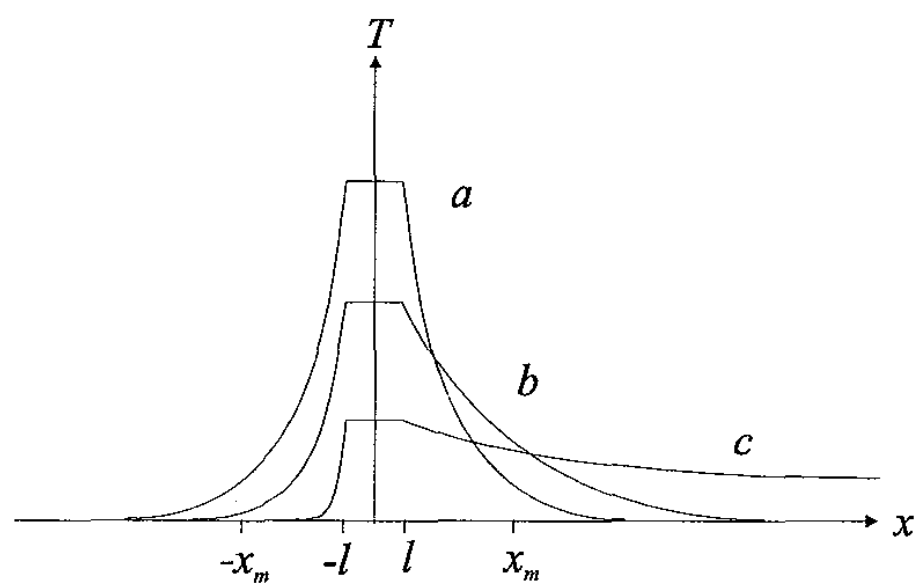

Fig. 6. Temperature distributions in the middle of the channel as a function of the position $x$. The heater extends from $x=-l$ to $x=l . x_{m}$ denote positions of temperature sensors. The curves characterize three typical flow velocities: $a: v=0 . b: v$ is small enough to allow heat to diffuse to the downstream temperature sensor. $c: v$ is too large to allow heat to diffuse to the downstream temperature sensor (Elwenspoek 1994).

Calorimetric flow sensors have an output, which is characterized by a linear dependency on the flow rate at small flow, a maximum and a decline (Johnson 1987, Komiya 1988, Lammerink 1993, Lyons $1998^{2}$, de Bree 1995, 1997, 1998, Ashauer 1998). A typical example is shown in fig. 7. These data are obtained with Lammerink's flow sensors (Lammerink 1993), for water flow. Geometric details are given in the figure caption. The temperature is measured via the electrical resistance of a chromium-gold thin film on top of a silicon nitride carrier. The chromium is necessary for the adhesion of gold to the silicon nitride, but has the disturbing property that it forms an alloy with the gold which has a much smaller temperature dependence of the resistivity than gold itself. The slow diffusion process gives additionally rise to a drift in the resistivity of the material. Sensors using this type of material must be "burned in" (de Bree 1997).

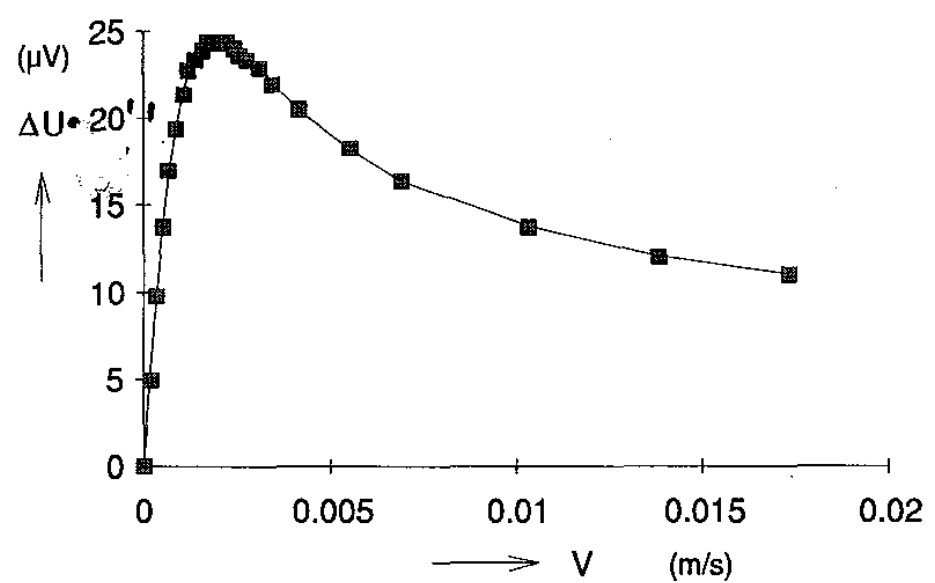

Fig. 7. Flow-sensor output with water as liquid. With a flow channel of $1000 \mu \mathrm{m} \times 500 \mu \mathrm{m}$, a flow velocity $v=20$ $\mathrm{mm} / \mathrm{s}$ corresponds to a volume flow of $600 \mu \mathrm{l} / \mathrm{min}$. For water: $\kappa=0.6 \mathrm{~W} / \mathrm{mK}, D=1.410^{-7} \mathrm{~m}^{2} / \mathrm{s}$. The distance between the temperature sensors was $2 \mathrm{~mm}$ (Lammerink 1993)

We found earlier than anemometers have a sensor characteristic, which contains the square root of the flow velocity. The physical reason is the boundary layer that builds up on walls and heated elements. In calorimetric

\footnotetext{
${ }^{2}$ Unfortunately, Lyons et al. are very reluctant to give any details of their geometry. Judging from their SEMimages, the temperature sensors are at a distance of less than $100 \mathrm{~mm}$, with chip size probably in the mm range
} 
flow sensors the temperature sensing elements are very close to each other, and the heat transport from the heater to the temperature sensors is not dominated by the boundary layer. The condition for the $\sqrt{U}$-characteristic is that $\delta \ll l$, in agreement with the anemometers described in the literature. Calorimetric flow sensors are designed such that the distance between the temperature sensors and the heater is very short, ranging from $1 \mathrm{~mm}$ (Lammerink 1993) down to $10 \mu \mathrm{m}$ (Ashauer 1998). Analysis shows that the linear regime is characterized by $\delta>l$. As an example, Ashauer et al. (1998) place heater and temperature sensors at a distance in a range of $l=10-150 \mu \mathrm{m}$, on a chip with a streamwise length of at least $1 \mathrm{~mm}$ (they only give the size of the membranes but not of the chip in the flow). They are using water, the plate length is $1 \mathrm{~mm}$ at least, and within a range of $\pm 4 \mathrm{~mm} / \mathrm{s}$ for the flow velocity, we find $\delta_{\min } \sim 500 \mu \mathrm{m} \gg l$.

Thus, in the regime of linear dependency of the temperature difference on the flow the Reynolds number is much smaller than 1. We have shown elsewhere (Elwenspoek and Lammerink 1999) that the relation $\Delta T \sim U$ results from a simple perturbation approach from the basic heat transfer equations.

The maximum of the curve is roughly given by the condition $\delta_{t h} \sim l$ with $\delta_{t h}$ the thermal boundary layer and $l$ the distance between heater and temperature sensor. We have approximately

$$
\delta_{t h} \approx \frac{\delta}{\operatorname{Pr}^{1 / 3}}
$$

or (Landau 1974)

$$
\operatorname{Re}^{m} \approx \operatorname{Pr}^{2 / 3}
$$

at the maximum. Interestingly a very similar equation can be derived (Elwenspoek 1994). If we assume that the maximum occurs in the case that the flow is too large that no heat can reach the upstream temperature sensor by diffusion, we may compare the diffusion time and the flow time:

$$
\frac{l^{2}}{2 D_{T}} \approx \frac{l}{U_{\max }}
$$

which leads to

$$
\frac{U_{\max } l}{2 D_{r}} \approx 1
$$

Here $U_{\max }$ is the flow velocity which corresponds to the maximum signal. (12) is essentially the same as (11) if $\mathrm{Pr}=1$ (except the factor 2 , which we grant for the estimates of order of magnitude). Since normally for gases $\operatorname{Pr} \approx 1,(12)$ and (11) can only be distinguished for fluids with Pr far from 1 (e.g. glycerol or mercury).

(11) corresponds quite satisfactory with the experimental results published so far, as seen in table 1 . We see a somewhat better correspondence with (11) than with (12).

\begin{tabular}{|l|l|l|l|l|l|l|l|}
\hline Author & fluid & $v\left(\mathrm{~cm}^{2} / \mathbf{s}\right)$ & $D_{r}\left(\mathrm{~cm}^{2} / \mathbf{s}\right)$ & $\operatorname{Pr}$ & $U_{\max }(\mathbf{c m} / \mathbf{s})$ & $l(\mathrm{~cm})$ & $\mathbf{R e}^{\mathrm{m}}$ \\
\hline $\begin{array}{l}\text { Lammerink } \\
(1993)\end{array}$ & water & 0.01 & 0.0015 & 6.7 & 0.1 & 0.1 & 1 \\
\hline Yang (1992) & water & 0.01 & 0.0015 & 6.7 & 0.2 & 0.15 & 3 \\
\hline $\begin{array}{l}\text { de Bree } \\
(1998)\end{array}$ & air & 0.15 & 0.15 & 1 & 10 & 0.01 & 0.7 \\
\hline
\end{tabular}

Table 1. Comparison of experimental results for the fluid velocity at maximum signal with eq. (11).

Lammerink (1993) has proposed a model to describe the temperature distribution around a heater in the presence of fluid flow. This model is essentially the same as the one given by Komiya (1988). The idea is to describe the heat transport from the hot element to the fluid and to the wall of the duct by lumped elements. The crucial assumption is that from the center of the duct upstream and downstream heat flows to the walls as if there 
were a heater at temperature $T$. The resulting differential equation is of the form (Komiya 1988, Hohenstatt 1990, Lammerink 1993),

$$
D_{\vartheta} \frac{\mathrm{d}^{2} T}{\mathrm{dx}^{2}}+U_{x} \frac{\mathrm{d} T}{\mathrm{dx}}-\beta T+s=0
$$

with s representing the strength of the heat source and $\beta$ is a parameter depending on the geometry of the duct. Lammerink gives a solution of (12) which is

upstream: $T(x)=T_{0} \exp \left\{\gamma_{2}(x-L)\right\}$

down stream: $T(x)=T_{0} \exp \left\{\gamma_{1}(x+L)\right\}$

with

$$
\gamma_{1,2}=\frac{1}{2 D_{T}}\left\{U \pm \sqrt{U^{2}+4 D_{T}{ }^{2} / h^{2}}\right\}
$$

$T_{0}$ is the temperature rise of the wire, $L=$ width of the heater and $G=2 w / h$ (width and half height of the channel). It is assumed that the heater and the temperature sensors are located in the center of the duct. The temperature difference between position symmetrically downstream and upstream of the heater indeed has a qualitative dependency as the experimental results shown in fig. 7 , but a quantitative comparison cannot be made. In this model, the maximum of the curve is given by the geometry of the duct and does not correspond to experimental results. The most important flaw of the model is the term $\beta T$ in (12), which causes a divergence of the heat flow where there are no heat sources.

\section{TIME OF FLIGHT SENSORS}

Calorimetric flow sensors can be operated in a dynamic mode. A heat pulse from the heating element will be transported by the convection to the downstream sensor. The pulse will deform by the velocity profile (fig. 8) and broaden at the same time by heat diffusion (fig. 9). Operated in this way, a pure time signal is measured, consequently only the velocity of the streaming fluid is measured, independent on the type of fluid. Since the heat transport by diffusion is important for the calorimetric flow sensor, in a static mode the sensor works in a different way for different fluids. The distortion of the signal by diffusion is serious only at small flow velocities. Roughly we can say that if the dispersion of the heat pulse is of the order of the distance between sensor and heater, the measurement of the time of flight becomes inaccurate. This means for flow velocities smaller than $U_{c}=D_{r} / l$ the signal tends to be too broad to be useful. But this relation approximately determines the maximum in the $\Delta T$ - $U$ curves, see (12). This means that calorimetric flow sensors operate optimal at velocities below $U_{c}$ and in the time of flight mode, there the optimal regime of operation starts. A discussion of this point can be found in Yang (1992) and in Ashauer (1998)

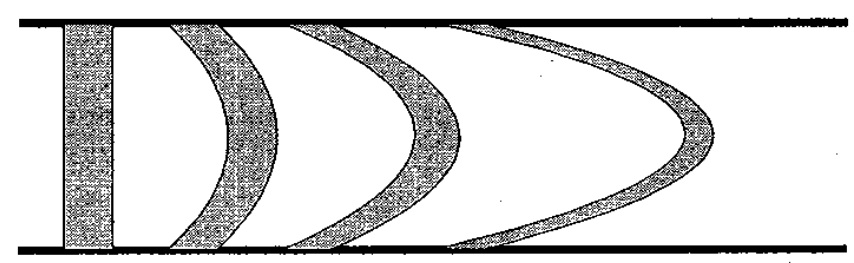

Fig. 8. Schematic of a heat pulse distortion in a flow with parabolic flow profile 


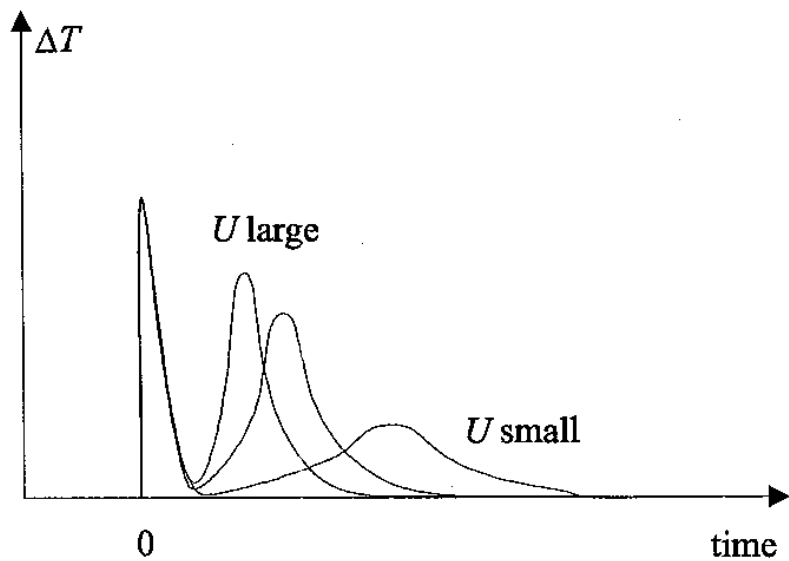

Fig.9. Schematic of a pulse distortion by dispersion in a time of flight flow sensor. A heat pulse is fed to the fluid at $t=0$. For fast flowing fluids the pulse comes quickly to the temperature sensor located downstream, and has no time to disperse. Small flow rates lead to broad, less intense and late pulses

\section{COMBINATION OF SENSOR PRINCIPLES}

A new configuration of combined heater/sensor elements has been proposed recently by de Bree et al. (1998), shown in fig. 8, with a SEM image in fig. 9. This instrument can be used as both: a calorimetric flow sensor, where the temperature difference of the two elements is measured at small flow rates and as an anemometer at larger flow, where the sum of the temperature of both elements is measured. The calorimetric sensor is much more sensitive than the anemometer at small velocities, but at larger velocity the anemometer becomes more advantageous.

Experimental results are shown in fig. 12.

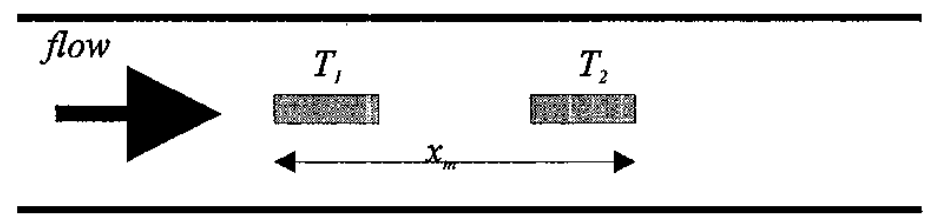

Fig. 10. Two element calorimetric flow sensor

Lammerink et al (1995) fed an AC-current through a three-element (sensor-heater-sensor) calorimetric flow sensor (fig, 4). Feeding a time varying signal to the heater leads to a mean temperature elevation of the heater plus a time varying component. If both is measured simultaneously, the mean temperature difference of the sensors upstream and downstream, and the time delay of the temperature with respect to the AC-heater current, more information than just the flow can be determined because materials properties of the fluid and the flow velocity influence heat transfer and time of flight in a different way. Lammerink (1995) and van Kuijk (1995) demonstrated that it is possible to extract flow and concentration of a $\mathrm{He}-\mathrm{N}_{2}$ mixture from the time dependent signal using a neural network. This technique leads to a remarkable precision $(0.5 \%$ for flow and concentration). 


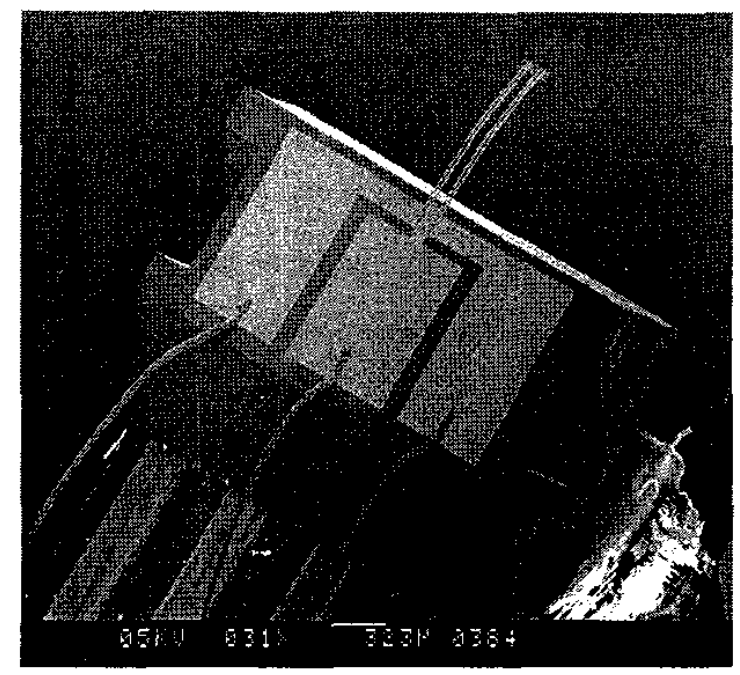

Figure 11. The $\mu$-flown, used as a bi-directional flow sensor.

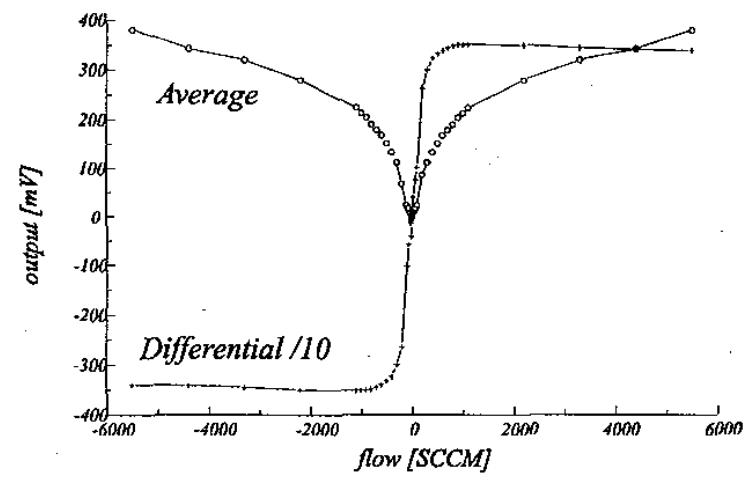

Fig. 12. Differential and common output signal of the sensor. Solid line: sum of signals, dashed line difference (de Bree 1998)

\section{REFERENCES}

Ashauer, M., H.Glosch, F.Hedrich,. N.Hey, H.Sandmaier, W.Lang, "Thermal flow sensor for liquids and gases", Proc. MEMS, Heidelberg 1998, p. 351-355

Boillat, M.A., A.J. van der Wiel, A.C. Hoogerwerf, N.F. de Rooij, "A differential pressure liquid flow

de Bree H.-E., Leussink P., Korthorst T., Jansen H., Lammerink T., Elwenspoek M., "The $\mu$-flown, a novel device measuring acoustical flows", Proc. Transducers 1995, Vol. 1, pp. 536-539

de Bree H.-E., Leussink P., Korthorst T., Jansen H., Lammerink T., Elwenspoek M., "The $\mu$-flown, a novel device measuring acoustical flows", Sensors and Actuators A, 54 (1996) 552-557

de Bree, H.-E., "The microflown", PhD Thesis, University of Twente (1997)

de Bree, H.-E., H.V. Jansen, T.J.S Lammerink, G.J.M. Krijnen, M. Elwenspoek, "Bi directional fast flow sensor with large dynamic range", Proc. MME '98, Ulvic (Norway) June 3-5, 1998, p.194-197

de Bree, H.-E., W.F. Druyvestyn, E. Berenschot, M. Elwenspoek, "Three-dimensional sound intensity measurements using microflown particle velocity sensors, Proc. MEMS, Orlando, Fl., USA, Jan. 1999, p. $124-129$ 
Ebefors, T., E. Kälvesten, G. Stemme, "Three dimensional silicon triple-hot-wire anemometer based on polyimide joints", Proc. MEMS '98, Heidelberg, Germany, January 25-29, 1998, pp. 93-98.

Elwenspoek M.C., T.S.J. Lammerink, R. Miyake, J.H.J. Fluitman, "Towards integrated micro liquid handling systems", J. Micromechanics Microeng. 4, (1994) 227-245.

Elwenspoek, M. and T.S.J. Lammerink, Eurosensors 13, 13-15 September, 1999, The Hague, The Netherlands

Elwenspoek M. and R. Wiegerink, Principles of Mechanical Microsensors, Springer (Heidelberg 2000) (in press)

Fingerson L.M., F. Freymuth, Thermal Anemometers, in: Fluid mechanics measurements, ed.R.J. Goldstein, Taylor \& Francis, Bristol 1996), pp. 115ff.

Funk, J., G. Wachutka, H. Baltes, "Scaling laws for optimised design of integrated thermopiles", Proc. Transducers, Yokohama, Japan, June 1993, p. 742-745

Hohenstatt, M. "Thermal mass flow meters", in: J. Scholz and T. Ricolfi (eds.) Sensors, a Comprehensive Survey, Vol 4. VZH, Weinheim 1990, Ch.9, pp. 325-330

Huysing, J.H., "monolithic flow sensors, a survey", Proc. Vaste Stof Sensoren conf. Delft, The Netherlands (1980) 39-48

Jiang, F. Y.C. Tai, C.H.Ho, R. Karan, M. Garstenauer, "Theoretical and experimental studies of the micromachined hot-wire anemometer, IEDM, San Fransico, 1994, p. 139-142

Jiang, F. Y.C. Tai, J.B. Huang, C.H. Ho, "Polysilicon structures for shear stress sensors", Digest IEEE TENCON'95, Hong Kong, Nove. 1995,

Jiang, F.-K., Tai, Y.-C., Gupta, B., Goodman, R., Tung, S., Huang, J., Ho, C.-M., "A surface micromachined Shear stress Imager", Proc. MEMS 1996, pp. 110-115

Kälvesten, E. Pressure and wall shear stress sensors for turbulence measurements, PhD thesis, KTH, Stockholm, Sweden 1996

Kälvesten, E., C. Vieider, L. Löfdahl, G. Stemme, "An integrated pressure-flow sensor for correlation measurements in turbulent gas flows", Sensors and Actuators A 52 (1996) 51-58

Kälvesten, E., L. Löfdahl, G. Stemme, "A small-size silicon microphone for measurements in turbulent gas flows", Sensors and Actuators A, 46 (1994a) 103-108

Kälvesten, E., L. Löfdahl, G. Stemme, "Small piezoresistive silicon microphones specially designed for the characterisation of turbulent gas flows", Sensors and Actuators A, 46 (1994b) 151-155

King, L.V. "On the convection of heat from small cylinders in a stream of fluid: Determination of the convection constants of small platinum wires with application to hot wire anemometry", Proc. Roy. Soc. London A, 90 (1914) 563-570

Kohl, F., A. Jachimowicz, J. Steurer, R. Glatz, J, Kuttner, D. Biacovsky, F. Olcaytug, G. Urban: "A micromachined flow sensor for liquid and gaseous fluids", Sensors and Actuators A, 41, (1994), 293 - 299.

Komiya, K, F. Higuchi, K. Ohtani, "Characteristic of a thermal gas flowmeter", Rev. Sci. Instrum. 59 (1988) 477-479

Kuttner, H., G. Urban, A. Jachimowicz, F. Kohl, F. Olcaytug, P. Goiser: "Microminiaturized Thermistor Arrays for Temperature Gradient, Flow and Perfusion Measurements", Sensors and Actuators A, 25 - 27 (1991), 641 -645 .

Lammerink, T.S.J., F. Dijkstra, Z. Houkes, J. van Kuijk, "Intelligent gas-mixture flow sensor", Sensors and Actuators 46-47 (1995) 380-384

Lammerink, T.S.J., N.R.Tas, M.Elwenspoek, J.H.J.Fluitman, "Micro-liquid flow sensor", Sensors Actuators 37-38 (1993) 45-50

Landau, L.D, E.M. Lifshitz, Hydrodynamik, Akademie Verlag Berlin 1974, pp.165ff.

Löfdahl, L., G. Stemme, B. Johansson, "Silicon based flow sensors used for mean velocity and turbulence measurements", Experiments in Fluids 12 (1992) 270-276

Lyons, C. A. Friedberger, W. Welser, G. Müller, G. Krötz, R. Rasing, "A high-speed mass flow sensor with heated silicon carbide bridges", Proc. MEMS, Heidelberg, Germany 1998, p. 356-360

Mayer, F. G. Salis, J. Funk, O. Paul, H. Baltes, "Scaling of thermal CMOS gas flow microsensors: experiment and simulation", Proc. MEMS, San Diego, CA, USA 1996, p. 116-121

Moser, D., R. Lenggenhager, H. Baltes, "Silicon Gas flow sensors using industrial CMOS and bipolar technology", Sensors and Actuators A 25, (1991)

Rehn, L.A., R.W. Tarpley, K.C. Wiemer, K.M. Durham, "Dual-element, solid state fluid flow sensor", $S A E$ Trans. 89 (1980) 705-710

Sanchez, S., M. Elwenspoek, C. Gui, M. de Nivelle, R. de Vries, P. de Korte, M. Bruijn, J. Wijnbergen, W. Michalke, E. Steinbeiss, T. Heidenblut, B. Schwierzi, "A high $\mathrm{T}_{\mathrm{c}}$ superconductor bolometer on a silicon nitride membrane", Proc. MEMS, Nagoya, Japan, January 26-30, 1997, p 506-511 
Stemme, G., " A monolithic gas flow sensors with polyimide as thermal insulator", IEEE Trans. Electron Devices, ED-33 (1986) 1470-1474

Stephan, C.H., M. Zanini, "A micromachined, silicon mass-air-flow sensor for automotive applications", Proc. Transducers, San Francisco, USA, 1991, p. 30-33.

Swart, N. A. Nathan, M. Shams, M. Parameswaran, "Numerical optimisation of flow-rate microsensors suing circuit simulation methods", Proc. Transducers, San Francisco, USA 1991, p. $26-29$

Tabata, O., H. Inagaki and I. Igarashi, "Monolithic pressure-flow sensor", IEEE Trans. Electron Devices, ED 34 (1987) 2456-2462

Urban, G., A. Jachimowicz, F. Kohl, H. Kuttner, F. Olcaytug, P. Goiser, O. Prohaska: "High resolution thinfilm temperature sensor arrays for medical applications", Sensors and Actuators A, 22 (1990), 650 - 654.

Van der Wiel, A.J., C. Linder and N.F. de Rooij, "A liquid velocity sensor based on the hot-wire principle", Sensors and Actuators A, 37-38 (1993) 693-697

van Heerwaarden, A.P., P.M.Sarro, "Thermal sensors based on the Seebeck effect", Sensors and Actuators, 10 (1986) 321-346

van Kuijk, J., T.S.J. Lammerink, H.-E. de Bree, M. Elwenspoek, J,H.J. Fluitman, "Multi parameter detection in fluid flows", Sensors and Actuators, A 46-47 (1995) 369-372

van Oudhuisden, B.W., "Integrated flow friction sensor", Sensors and Actuators, 15 (1988) 135-144

Van Oudhuisden, B.W., A.W. van Herwaarden, "High-sensitivity 2-D flow sensors with an etched thermal isolation structure", Sensors and Actuators A 21-23 (1990a) 425-430

Van Oudhuisden, B.W., J.H. Huysing, "An electronic wind meter based on a silicon flow sensor, Sensors and Actuators A 21-23 (1990b) 420-424

van Putten, A.F.P., "An integrated silicon double bridge anemometer", Sensors and Actuators 4 (1983) 387-396

van Putten, A.F.P., S. Middelhoek, "Integrated silicon anemometer", Electron. Lett. 10 (1974) 425-426

van Riet, R.W.M., J.H. Huysing, "Integrated direction sensitive flow meter", Electron. Lett. 12 (1976) 647-648

Wachutka, G., R. Lenggenhager, D. Moser, H. Baltes, "Analytical 2D-model of CMOS micromachined gas flow sensors", Proc. Transducers 1991, pp. 22-25

Yang, C., H. Soeberg: "Monolithic flow sensor for measuring millilitre per minute liquid flow", Sensors and Actuators A, 33 (1992), 143 - 153. 